\title{
Externalidades territoriales de la gobernanza financiera global
}

\author{
Antonio Daber. Pontificia Universidad Católica de Chile; Santiago, Chile.
}

RESUMEN | Las políticas públicas de los organismos estatales y supranacionales de la gobernanza financiera global orientadas a superar la crisis, implican una reacción de financiarización pública y de geopolítica financiarizada, instrumentos estos de dominio de las economías más desarrolladas y/o de competencia entre ellas. Esas políticas, preferentemente las monetarias y cambiarias, generan externalidades o "subproductos" territoriales y urbanos a escala tanto plurinacional como local. Se traducen en una nueva geografía de las tasas de interés y sus diferenciales, reorientando los flujos de capital a distintos países; y en una nueva geografía de los tipos de cambio y sus paridades, expresión de la pugna competitiva entre regiones y naciones. Al mismo tiempo, externalizan muchos de los costos de la crisis a los gobiernos y sociedades de las naciones emergentes, aportando sustentabilidad a unos pocos países (los más fuertes) a cambio de vulnerabilidad para muchos otros (los más débiles), en una inequitativa redistribución socioterritorial de los costos y beneficios de los ajustes que promueven. La arquitectura global del territorio resultante de tales políticas macroeconómicas demuestra su no neutralidad geográfica ni social.

PALABRAS ClAVE | políticas públicas, gobernanza financiera, financiarización pública, geografía monetaria y cambiaria, crisis.

ABSTRACT | Public policies of state and supranational organisms involved in global financial governance aimed at overcoming crisis imply a reaction based on public financing and financed geopolitics, instruments controlled by the most developed economies andlor competition among them. Such post-crisis policies, mainly referring to monetary and exchange rate policies, produce territorial and urban externalities or 'sub-products' at pluri-national and local scales, and translate into a new geography of interest rates and their corresponding differentials. This induces a reorientation of capital flows to different countries, a new geography of exchange rates and their corresponding parities, and expressions of competitive rivalry among regions and nations. These policies, which externalize many crisis costs to governments and societies of emerging nations are sustainable only for a few countries (typically the most developed ones), for the vulnerability of many others (mainly the weakest nations), in the context of unequal socio-territorial redistribution of the costs and benefits of such adjustments. The global territorial architecture resulting from these macroeconomic policies shows the non-neutral geographic and social nature of their impacts.

KEY WORDs | public policies, financial governance, public financing, monetary and exchange rate geography, crisis.

Recibido el 21 de mayo de 2014, aprobado el 20 de febrero de 2015

E-mail: adaher@uc.cl 


\section{Introducción}

La cuestión central tratada en este artículo puede expresarse en los siguientes términos: las políticas anti y poscrisis -económicas y, particularmente, financieras-, sobre todo de las grandes potencias y organismos internacionales, generan externalidades territoriales y urbanas a escala tanto plurinacional como local. Contagian y endosan los costos de la crisis, detonando a la vez múltiples inequidades sociales, desde el desempleo multitudinario hasta la emergencia de nuevas pobrezas, con lo que alteran la geografía económica, social e incluso política. Asimismo, es posible conjeturar que si la crisis fue una resultante, en significativa medida, de la financiarización privada y la desregulación, las políticas públicas aplicadas para confrontarla -preferentemente las monetarias, fiscales y cambiarias- representan ahora intervenciones de organismos estatales y supranacionales, equivalentes a una auténtica reacción de "financiarización pública" a escala nacional, internacional y global.

Si bien este artículo analiza los efectos territoriales de las políticas macro en el contexto de la reciente crisis global, donde tales efectos se hacen más evidentes, su tesis central -es decir, la no neutralidad espacial de dichas políticas- es aplicable igualmente en otros contextos históricos y en escenarios económicos diferentes.

Las medidas de políticas supranacionales y locales para afrontar los riesgos recurrentes tanto de default soberanos como de "precipicios fiscales" (fiscal cliffs) en países tan extremadamente polares como Chipre y los Estados Unidos (EE.UU.), pero también intermedios, como Grecia y España; la amenaza de deserción de algunas naciones de la Eurozona por las tensiones norte-sur, y los intereses manifiestos de otras por una más plena integración -en rigor, mayor regulación- vía unión fiscal y bancaria, para mejor controlar así a los "países periféricos"; las más o menos tácitas o declaradas guerras de divisas entre Japón, China y los EE.uU. con el propósito de favorecer su competitividad; y, en general, la confrontación de regímenes cambiarios y políticas monetarias junto a la emergencia de monedas alternativas de refugio propias de países en desarrollo, son algunas de las manifestaciones de la poscrisis y de las políticas públicas nacionales, internacionales y supranacionales puestas en juego.

¿Qué expresiones y efectos geográficos y urbanos conllevan estas políticas macro? En general, sus "subproductos" son tanto territoriales como sociales; por ejemplo: i) se registran en una nueva geografía de las tasas de interés y sus diferenciales, reorientando los flujos de capital y las inversiones a distintos países; ii) se "mapean" en una nueva geografía de los tipos de cambio y sus paridades - o, si se prefiere, disparidades-, expresión de la pugna competitiva entre regiones y naciones, incentivando alternativamente la reasignación de recursos a sectores exportadores o importadores y a sus respectivos territorios provinciales o estaduales; iii) se manifiestan en una nueva geografía del riesgo-país, tanto de los riesgos subnacionales y específicamente locales -posibles default urbanos o municipales (Martin, 2011; Perlo, 2011)- como supranacionales - por efectos barrio, dominó, manada (Esquivel \& Larraín, 1997; Hernández \& Valdés, 2001)-, y también en cartografías reales del contagio territorial de las crisis; iv) se grafican en una geografía alternativa de la "economía real", con cambios sectoriales y territoriales en la producción, y con procesos urbanos y metropolitanos de desindustrialización y reindustrialización, y también con 
volatilidad en las "regiones-commodities" o "territorios de exportación"; y v) sobre todo y más dramáticamente, se acusan en una "otra" geografía de los mercados laborales, de migraciones forzadas, paro y cesantía, depreciación salarial, pérdida de beneficios sociales y emergencia de pobrezas inéditas, rurales y urbanas, centrales y periféricas, en el mundo desarrollado y en desarrollo.

En el contexto iniciado en la crisis inmobiliaria y financiera detonada en 2008 en los Estados Unidos, sin duda la más global en el espacio y una de las más perseverantes en el tiempo, algunos titulares de la literatura especializada ilustran incisivamente sus consecuencias y prefiguran a la vez el escenario poscrisis: un conocido autor escribía sobre la crisis, aludiéndola con cierto pesimismo, en un libro denominado De vuelta a la economía de la gran depresión (Krugman, 2010). Roubini y Mihm (2010), el primero de ellos reconocido como uno de los "profetas" de esa crisis, con un tono de perplejidad si acaso no de desesperanza, planteaban la pregunta: “¿Cómo salimos de esta?”. Otros autores, como Reinhart y Rogoff (2011), denunciaban en su título que la actual crisis, como muchas precedentes, respondía a "Ocho siglos de necedad financiera". Y Stiglitz (2010), acusando responsabilidades, escribía sobre las causas de la crisis bajo la denominación "El libre mercado y el hundimiento de la economía mundial".

En este convulsionado escenario y complejo contexto, Aglietta (2012) planteaba una cuestión más específica, pero fundamental: ¿por qué la Eurozona se constituye como el nuevo centro de una crisis cuyo origen -las hipotecas subprime- era norteamericano? Esta interrogante refería inequívocamente al contagio y difusión (Aalbers, 2009), pero a la vez innegablemente a una geografía de la crisis. Se trataría, según Harvey (2011), del tránsito de crisis localizadas a crisis globales. En efecto, llama la atención e incluso sorprende que a partir de un "subsector" inmobiliario particular -el subprime, relacionado con la vivienda social para afroamericanos, hispanos y otras minorías étnicas y pobres, localizado solo en "ciertos" barrios y áreas de "algunas" ciudades estadounidenses-, la crisis se expandiera con celeridad a la totalidad del sector financiero y, mediante él, a la economía mundial en su conjunto. Esta propagación motivaría a Dymski (2012) a rotularla asertivamente "desde los márgenes de la ciudad al corazón de las finanzas globales" (p. 161). Coincidentemente, Saskia Sassen (2012), refiriéndose a la securitización como medio de inducción y propulsión del contagio en tiempo real de la crisis, la describía gráficamente en los siguientes términos: "When local housing becomes an electronic instrument" (p. 74).

En consecuencia, "las raíces urbanas de las crisis financieras" intuidas por Harvey (2012a, p. 321; y 2012b, p xiii), así como, más enfáticamente, la "centralidad de las ciudades en la crisis", reconocida por Aalbers (2012, p. 6), remiten a la exhortación de que "las conexiones entre urbanización, acumulación de capital y financiarización de las crisis merecen un cuidadosa análisis" (Harvey, 2011, p. 137), y a la vez aluden a la necesidad de expansión geográfica del capital global (Sassen, 2012). La relación históricamente recurrente entre el sector inmobiliario y las crisis económicas (Daher, 2013a) confirma tal imbricación, y la creciente financiarización urbana, no solo inmobiliaria (Daher, 2013b), la hace cada vez más indisociable, potenciando su globalización. 
En efecto, la mayoritaria y ascendente urbanización demográfica, económica y especialmente financiera, hace imposible desvincular los ciclos económicos de las dinámicas urbanas y, más aún, metropolitanas, estrecha y dialécticamente interrelacionadas. Jones y Ward (2002) recordaban que varios estudios constataban que las ciudades son tanto el lugar de variadas crisis como el de su solución. Quizás por esta patente realidad, Harvey (2011) formula una interrogante de indesmentible actualidad: "¿la urbanización de China es la fuente primaria de estabilización del capitalismo mundial?” (p. 142), relevando como factor causal a las ciudades; y probablemente por razones análogas, Aalbers (2012) se pregunta por las "ciudades pos subprime" (p. 293), en directa alusión a los impactos urbanos de la crisis.

La relación entre ciclos económicos y dinámicas urbanas en la complejidad de la mundialización lleva a González (2005) -siguiendo a Brenner y a varios autores- a recordar que los cambios económicos y territoriales más recientes corresponden a un "reescalamiento" de procesos socioeconómicos y políticos, más allá de una simple oposición entre las tradicionales categorías polares de lo local y lo global. De hecho, de acuerdo con Brenner (2004), las instituciones supranacionales y transfronterizas cumplen una función reguladora multiestatal y de gobernanza multinivel, facilitando el libre comercio y los flujos de inversión. El mismo autor sostiene que instituciones como el Banco Mundial (BM), el Grupo de los Ocho (G8), la Unión Europea y el Foro Económico Mundial, entre otras, serían las instancias promotoras y mediadoras máximas de los procesos de "reescalamiento" de los estados nacionales en relación con los ámbitos económicos y, sobre todo, financieros. Es oportuno aclarar desde ya que, en esta dialéctica escalar, las regiones subnacionales obviamente no constituyen economías autónomas, como también que la transferencia de efectos entre países y entre regiones y ciudades no necesariamente es unidireccional ni simétrica, y puede responder a carriers distintos, no siempre intermediados por la escala nacional. Con ello se hace evidente que los espacios económicos cada vez más sobrepasan los límites político-administrativos internacionales y subnacionales.

Por cierto, tales cuestiones conciernen también y especialmente a países con economías emergentes, no pocas veces hiperurbanizados, y cuyas regiones y áreas metropolitanas acusan los impactos de las crisis globales (Méndez, 2013; Noyola, De Mattos \& Orellana, 2013), no solo por el contagio inducido a través de los carriers financieros públicos y privados, sino muy fuertemente vía comercio internacional y precios de sus commodities. A esta doble vulnerabilidad y a sus implicaciones sociales y ambientales se suma el hecho de que sus políticas públicas suelen resultar reactivas y marginales frente a las propias de las grandes potencias.

En los meses de redacción final de este texto (inicios de 2014), la Reserva Federal estadounidense comenzaba el tapering o disminución progresiva de su programa de "relajamiento cuantitativo" nominado QE3 (tercer Quantitative Easing), consistente en una oferta de adquisición de bonos y activos hipotecarios por US\$ 85.000 millones mensuales para estimular el empleo, el gasto y el crecimiento, aunque conservando su tasa de interés en mínimos históricos, al igual que el Reino Unido, Japón y varios países europeos. El Banco Central Europeo con anterioridad había experimentado, por su parte, el programa de compras monetarias directas омт (Outright Monetary Transactions), además de las iniciativas del Fondo Europeo 
de Estabilidad Financiera (EFSF) y del Mecanismo Europeo de Estabilidad (ESM). Ahora, en la primavera europea de 2014, los mercados esperaban una política monetaria expansiva para evitar una eventual deflación y/o estagnación en ese continente. En uno y otro caso, antes, durante y después del QE3, y también en cada momento de inflexión de la política monetaria europea -o de las potencias asiáticas-, los bancos centrales de los demás países, sobre todo los emergentes y de economías menores, debieron actuar y actuaron reactivamente frente a una cadena de procesos: la apreciación o devaluación de sus monedas, los ingresos y egresos de capitales foráneos, las alteraciones en sus balanzas comerciales y de pagos, los cambios en sus tasas de crecimiento, los ajustes en sus mercados laborales y demás consecuencias sociales e incluso políticas. La contraparte territorial de estas políticas macroeconómicas transnacionales y nacionales y su "huella geográfica" constituyen el desafío de este artículo. Aunque las mismas ciertamente también generan externalidades o "subproductos" territoriales no solo en los períodos de crisis, durante estos los ajustes de política se extreman y, por ende, los impactos urbanos y regionales se hacen más críticos.

El texto a continuación se estructura en los siguientes apartados: Geografía de la financiarización pública; Políticas y zonas monetarias; Políticas cambiarias y geografía de divisas. Al inicio de cada sección se hace un breve enunciado de sus contenidos. Finalmente, el artículo se cierra con una sección de Conclusiones.

\section{Geografía de la financiarización pública}

En esta primera sección se recuerdan inicialmente algunos de los principales aportes a la discusión de temas que relacionan la finaciarización con el territorio, postulando desde el presunto "fin de la geografía" hasta la emergencia de una pretendida "nueva" geografía económica. Se reflexiona, además, sobre la supuesta ubicuidad y aespacialidad de la financiarización, aludiéndose también a la articulación entre la reciente crisis financiera y el territorio. A continuación se introduce preliminarmente al tema central, la financiarización pública vinculada a las políticas macroeconómicas tanto nacionales - principalmente monetarias y cambiarias- como de organismos supranacionales. Se examina la interacción recíproca de tales políticas con el mercado global de capitales y, tras este, con una relativa privatización en la gobernanza financiera global, con tensiones y sutiles equilibrios entre regulación y desregulación. Finalmente, se esbozan algunos alcances al problema del riesgo asociado tanto a la propia financiarización pública como a la nueva gobernanza financiera global, privatizada y supraestatizada a la vez; tal riesgo, se seńala, remite a las crisis y su contagio, atendidas la volatilidad espacial de los flujos de capitales y de los propios fondos soberanos, como asimismo la geografía variable de la deuda externa

En el contexto en el que países, regiones y continentes pujan por superar la crisis, pero también aún en medio de una "financiarización en crisis", es conveniente atender a la relación entre territorio y financiarización. Pareciera oportuno volver a preguntarse si la globalización financiera lleva al "fin de la geografía" (O’Brien, 1992; French, Leyshon \& Thrift, 2009). Y quizás en este punto -además de reflexionar si acaso surge "una nueva geografía económica" (como la intitula el 
Banco Mundial, 2008, o preguntarse, con Cuadrado-Roura (2014), si es tan "nueva" la nueva geografía económica, o con Sokol (2013, p. 501) si se avanza "hacia una newer geografía económica” - más bien corresponda restarle primacía al sector real y afirmar categóricamente que la geografía económica más auténticamente nueva es la financiera y, más precisamente, la "geografía de la financiarización".

Tal vez por esto Harvey (2011) vincula las nuevas geografías con los flujos espaciales de capital, localizables aún en medio de un mercado globalizado e instantáneo de capitales (Carriére-Swallow \& Claro, 2013); y ciertamente por observaciones similares, Bravo, García, Mies y Tapia (2003) analizan las consecuencias sectoriales y "regionales" de la heterogeneidad de la transmisión monetaria. Porque, en efecto y al contrario de lo aparentemente más obvio, la mayor y creciente movilidad y liquidez del capital ha producido evidentes y significativos cambios en el nivel regional (Corpataux \& Crevoisier, 2005), y el hiperdesarrollo de la industria de las finanzas ha tenido un impacto esperable no solo en la economía, también y por lo mismo en los territorios (Corpataux, Crevoisier \& Theurillat, 2009).

Aunque la financiarización maximiza la movilidad de capitales, no implica, sin embargo, su ubicuidad. Su "novedad" deriva de las innovaciones en la ingeniería financiera, no de una presunta "desterritorialización” (Daher, 2013b). De hecho, González (2005) enfatiza que este "nuevo capitalismo no es aespacial, sino todo lo contrario" (p. 2) y, recordando a Harvey (2000), alude a sus necesarios "anclajes espaciales" y a la continua construcción y reconstrucción de su geografía. Puede afirmarse aquí que la financiarización opera en la instantaneidad del "tiempo real" tanto como en la tangibilidad geográfica del "espacio real”, sin mutua exclusión. En otros términos, Dymsky (2009) plantea que "la globalización financiera no hace inmaterial a la geografía” (p. 267); y Lee, Clark, Pollard y Leyshon (2009), enfatizando la condición territorial, aluden a "un antes y un después de la crisis para la geografía financiera” (p. 723). Acertadamente Wainwright (2012) habla de "una geografía histórica de la securitización" (p. 102), refiriéndose a los canales de transferencia de la financiarización, respecto de los cuales cabe agregar que tan relevantes como los flujos son los lugares de orígenes y destinos, y los relaciona con el contagio -agreguemos, no solo financiero, también geográfico- de la crisis reciente. De hecho, otros autores, como French, Leyshon y Thrift (2009) la llegan a caracterizar, muy elocuentemente, como "una muy geográfica crisis" (p. 287). Así, consecuentemente, se podría responder a la cuestión planteada por Aalbers (2012), quien, con un tono de cierta perplejidad, se pregunta "por qué la desterritorialización ha sido tan lenta" (p. 137).

Así pues, si la financiarización es comprendida y definida, siguiendo a Corpataux y Crevoisier (2005), como la construcción de la movilidad / liquidez del capital, entonces puede verificarse que la financiarización pública no solo replica muchos de los instrumentos de la ingeniería financiera y no pocos de sus riesgos -hiperemisión, sobreendeudamiento externo público, déficit fiscal descontrolado-, sino que ella misma lidera habitualmente los flujos de capitales y sus reorientaciones geográficas, controlando legal y administrativamente su movilidad y regulando la mayor o menor liquidez en los mercados. 
Si bien la literatura especializada en geografía económica y la mayoría de sus estudios de casos se refieren principalmente y más a los flujos privados de capital, y menos y más marginalmente a las asignaciones fiscales, las inversiones de capitales estatales y las colocaciones internacionales de fondos soberanos, es evidente que sus efectos sectoriales y territoriales y, más aún, los de las propias políticas monetarias -que condicionan, junto a otros factores, la movilidad de los capitales privados-, tampoco resultan geográficamente neutros u homogéneos. En consecuencia, aunque indivisiblemente relacionadas, convergen dos aproximaciones a la geografía financiera: una, más conocida y difundida, relacionada con las finanzas privadas y el mercado; otra, tal vez menos explorada y divulgada, derivada de las finanzas públicas y las políticas macroeconómicas estatales, como también de las propias de organismos supranacionales.

Tales políticas públicas en el ámbito macroeconómico y financiero a escala nacional y global remiten necesariamente a problemas de regulación. Siguiendo a Gill (1995) en su concepto de disciplinary neoliberalism, Brenner y Theodore (2002a) destacan la contribución ordenadora regulatoria de instituciones como el Banco Mundial, el Fondo Monetario Internacional, la Organización Mundial de Comercio, la Unión Europea y el Tratado de Libre Comercio de América del Norte, es decir, de instancias supranacionales, en contraste con la desregulación creciente de los sistemas nacionales. En otro escrito, los mismos Brenner y Theodore (2002b) subrayan el rol de tales organizaciones en disciplinar los mercados de capitales de los países semiperiféricos y periféricos, transformándose así en agentes de un proyecto geopolítico y geoeconómico, dentro del cual aquellas desempeñarían un rol crecientemente hegemónico en el fomento de la desregulación, la movilidad de capitales, la liberalización del comercio y la commodification generalizada. ¿Contradicción en el rol disciplinario y a la vez desregulador de tales organismos transnacionales? No necesariamente, según Peck y Tickell (2002), pues se trataría de "una forma de metarregulación, un sistema de reglas que paradójicamente se define a sí mismo como una forma de antirregulación" (p. 53).

Por su parte, Sassen (2010), en relación con la globalización de los mercados financieros y el rol estratégico de los bancos centrales, señala que estos "se han convertido en el espacio institucional nacional en el que se instauran las políticas monetarias necesarias para promover el desarrollo del mercado global de capitales", agregando que, a su vez, "los ministerios de economía (o el Tesoro de los Estados Unidos) (...) han debido imponer ciertas políticas fiscales como parte de los requisitos que plantea la globalización económica" (p. 293). La autora agrega que "la formación del mercado global de capitales representa una concentración de poder con capacidad para influir en las políticas económicas de los gobiernos nacionales y, por extensión, en sus otras políticas", lo que se traduce en su capacidad de disciplinarlas, reduciendo incluso -a través de los instrumentos derivados- la "eficacia de las políticas estatales relacionadas con (la) tasa (de interés)", tal como el mercado de divisas puede debilitar "el poder gubernamental (...) para intervenir en el tipo de cambio" (pp. 312, 326, $327,328)$. Pone en relieve, además, la importancia del sector privado global y su "autoridad" en áreas como el arbitraje comercial internacional, el sistema de agencias de calificación crediticia y, en relación con el sector inmobiliario, la lex constructionis, 
todos campos de una nueva institucionalidad transnacional de regulación e incluso de "justicia" privadas, que implican un desplazamiento de la autoridad del sector público al privado en asuntos de gobierno de la economía global. Con todo, reconoce una tendencia a la convergencia entre los bancos centrales y el papel estratégico de los "organismos supranacionales, como el Fondo Monetario Internacional [en la] gobernabilidad no estatal del mercado global de capitales” (p. 329).

En tal escenario, se dan diversas variables explicativas de los flujos de capitales, de su sesgo y asignación territorial y, en distintos horizontes temporales, de su "volatilidad espacial" y migraciones geográficas. Entre ellas, los diferenciales internacionales (y, en algunos países, también subnacionales) de tasas de interés y tipos de cambio (incluidos los no oficiales, como el dólar "negro" o el blue argentino), que responden parcialmente a condiciones de mercado y fuertemente a políticas públicas, aunque también en ocasiones a acuerdos bancarios ilegales, como en los casos recientes de las tasas LIBOR (London Interbank Offered Rate) y EURIBOR (Euro Interbank Offered Rate). Y junto a tales factores, es necesario sumar otros, como el riesgo país, las restricciones a la libre circulación de divisas y la tributación (o su omisión, evasión o elusión en "paraísos fiscales”).

Por lo demás -como sucedió, por ejemplo, en 1997 en el sudeste asiático-, aquellos flujos pueden llegar a inducir burbujas especulativas locales, sobreapreciación de las monedas domésticas y pérdida de competitividad, amén de otros efectos; $y$, en extremo, crisis económicas que contaminan el vecindario internacional y luego se hacen más o menos globales. Son los riesgos de la invasión -más que de la inversión- de capitales y de los desequilibrios en la geografía de las tasas de interés que deben asumir coyunturalmente los países emergentes y sus circunstanciales "monedas refugio".

Ciertamente, la geografía financiera pública también se conforma y varía según sea la distribución territorial, en diversos países o "carteras" de países, de las colocaciones de los fondos soberanos, y la correspondiente distribución espacial de los deudores. Esto se traduce en regiones y países colocadores netos, no siempre los más desarrollados; y en territorios captadores netos, no siempre los de menor desarrollo (de hecho, China y Japón son los mayores inversionistas en bonos soberanos de los EE.UU.).

Por otra parte, en coyunturas de crisis, la geografía de la deuda externa pública detona muchas veces procesos de conversión territorial como correlato de la conversión de deuda, incluidos convenios financiero-ambientales como los debt for nature swaps, además de variados ajustes urbanos y regionales. Con una crisis generada por una inconmensurable financiarización privada, permitida y acompañada de una amplia desregulación, las reacciones antirrecesivas y procompetitividad manifiestas en las políticas estatales y supranacionales poscrisis constituyen, en síntesis, una "financiarización pública" con fuertes consecuencias sociales y territoriales, que induce a indagarlas bajo la aproximación conceptual de una geografía financiera estatal y supraestatal, y más aún, de una "geografía de la financiarización pública". Esta se configura, según se verá a continuación, expresando y "territorializando" variables como la política cambiaria, la monetaria y fiscal, con efectos agregados en los mercados financieros locales y globales y en sus respectivas geografías. 


\section{Políticas y zonas monetarias}

Esta sección está principal -aunque no exclusivamente- referida, dentro del ámbito más general de las políticas monetarias y sus efectos territoriales, a las denominadas "zonas monetarias", terminología que de suyo pone en relieve su sustrato geográfico. Complementariamente, y solo de modo muy preliminar, se esbozan algunas de sus implicaciones fiscales.

Un breve recuento introductorio permite reconocer los aportes sobre la "geografía de las transformaciones monetarias", realizados, entre otros por Leyshon (1997), y los estudios relacionados con "el dinero y el espacio económico" pormenorizados por Martin (1998) en una obra homónima. Precediéndolos, Mundell (1961) había investigado sobre la integración monetaria y su relación con la integración fiscal. Algunos de sus planteamientos conceptuales pueden ilustrarse con un caso concreto: un sugerente estudio del Banco Central de Chile corroboró que las regiones subnacionales con orientación industrial y comercial, comúnmente urbanas, son más sensibles, usando como indicador el desempleo, a los ajustes de política monetaria que las regiones mineras o agrícolas, tradicionalmente más rurales (Bravo et al., 2003). Tal conclusión, verificándose una desigual distribución territorial de los costos y beneficios de dichos ajustes, al menos cuestiona, si acaso no desecha, la pretendida neutralidad de ciertas políticas públicas macroeconómicas, y la supuesta virtud de su homogeneidad.

Retomando la evolución conceptual en estos tópicos, uno de los aportes teóricos más determinantes es de la autoría de McKinnon (1963), quien indagó acerca de las "zonas monetarias óptimas" (véase también Kennen, 1969; y Sala-i-Martin \& Sachs, 1991). Por cierto, el concepto de "integración” de Mundell ya alude a una cierta dimensión "espacial", la que se hace nítidamente explícita en el de "zona" monetaria de McKinnon, aún más si se lo asocia a una suerte de área o cuantía óptima.

Ahora bien, en una zona monetaria, una moneda "única" -como el euro- tendría, entre otros beneficios, la reducción de algunos costos de transacción, extremadamente significativa entre países que, como suele suceder con los colindantes o próximos de una misma región, comparten un gran comercio (Krugman, 2012), incidiendo así positivamente en su desarrollo intrazonal (Carriére-Swallow \& Claro, 2013). Como es sabido, más de la mitad del comercio internacional europeo es intrarregional. Además, en términos de riesgo, la moneda única constituiría una especie de garantía tácita (Huerta de Soto, 2012), otorgando más seguridad a inversores y acreedores. Sin embargo, y como paradoja, más adelante se verá que esta "garantía" se trastocó, en el caso europeo, en un crítico problema de endeudamiento, incluso soberano, y que la ventaja comercial intrazonal implicó una grave desventaja competitiva extrazonal para muchos países y regiones de la unión monetaria.

Las zonas monetarias, concebidas principalmente desde una perspectiva económica, ameritan ciertamente también una evaluación territorial. Algunas cuestiones en esta aproximación son fundamentales: ¡las divergencias regionales se reducen o, por el contrario, se acentúan en la intrazona? En otros términos, la moneda única ¿es proconvergencia? (Canova, 2001); la mayor integración económica asociada a la unidad monetaria ¿aporta en la superación de las disparidades interregionales? (Suárez-Villa \& Cuadrado Roura, 1993); ¿qué incidencia tienen las zonas 
monetarias en los desequilibrios territoriales? (Rodríguez-Pose \& Petrakos, 2004). Estas interrogantes adquieren especificidad e interés contingente en la experiencia de la Eurozona (Ezcurra, Pascual \& Rapún, 2003), sea que se consideren directamente indicadores territoriales, o bien que se evalúe indirectamente el impacto de la moneda única por sus efectos en la competitividad y el crecimiento económico de sus regiones (Rodil, 2008).

Antes y después de la reciente crisis europea, las respuestas a estas cuestiones son categóricas: luego del Tratado de Maastricht, cuando el euro fue introducido, las expectativas eran de convergencia, pero en realidad la unidad monetaria generó divergencias (Soros, 2012). Resultan evidentes sus efectos contrapuestos en el norte y en el sur, como asimismo las diferencias interregionales al interior de cada país y también entre ciudades (Huerta de Soto, 2012). Otro tanto sucedió, en términos de diferenciación geográfica, con la distribución sectorial y territorial de la inversión externa directa, tanto de empresas privadas como de consorcios estatales o mixtos: un ejemplo, entre otros estudios, es el análisis comparado de la inversión extranjera directa (IED) en las regiones españolas (Rodríguez, 2005), que confirma tal heterogeneidad y sus previsibles consecuencias económicas, laborales y sociales.

Durante la última crisis, Krugman (2012) verificó impactos diferenciales de la misma en las distintas zonas del continente. El mismo autor es aún más crítico al referirse a la caída de competitividad de los países sudeuropeos y al abultamiento de sus déficits comerciales. Lejos de una mayor convergencia, habría agudización de muchas divergencias. Huerta de Soto (2012) afirma que se habría desencadenado una "maciza redistribución de riqueza en toda la unión monetaria" (p. 16), en otras palabras, un auténtico "proceso de transferencia de riqueza" (p. 22). Por consideraciones como las anteriores, y siguiendo a Feldstein, Van Overtveldt (2012) sostiene que la unión monetaria induciría "más guerra que paz" al interior de Europa.

La política monetaria de los gobiernos europeos fue obviamente afectada, en términos de su independencia, por la introducción de la moneda única: "la política monetaria de la Unión puede ser muy apretada (ajustada) para la economía de un país miembro y muy floja (laxa) para otro", obligando a las autoridades nacionales a echar mano a políticas no monetarias para lograr ciertas compensaciones (Van Overtveldt, 2012, pp. 52-53).Complementariamente, el menor costo del crédito para las naciones del sur -dado su supuesto menor riesgo por la moneda única-alzó los precios de muchos bienes de capital y contribuyó a la inflación de burbujas inmobiliarias (Huerta de Soto, 2012), erosionando los fundamentos financieros de tales regiones y, paradójicamente, incrementando su riesgo (Martin \& Minns, 1995). Así pues, la convergencia en las tasas crediticias generó, como otra paradoja, divergencia económica entre las naciones fuertes y las débiles. La Eurozona se escindió entre naciones deudoras y acreedoras, y la integración se convirtió en desintegración (Soros, 2012).

En palabras de Harvey (2007), se desbaratan así las "alianzas de clase regionales" enturbiándose "las relaciones entre ellas" (p. 361). Por su parte, Sassen, en su sugerente Contrageografías de la globalización (2003) y en referencia a las "posibilidades emergentes de una nueva geografía política” (p. 87), llega a plantear el 
dilema “¿postnacional o desnacionalizado?” (p. 104) que, aun cuando formulado en alusión a otro contexto, resulta apropiado y oportuno a estas reflexiones.

De hecho, si bien los costos asociados a los déficits en algunos países pueden ser en parte externalizados a otros mediante inflación en ellos (Huerta de Soto, 2012), ciertamente el "efecto Estado" acrecentó las divergencias, beneficiando a las regiones del centro de la Unión Europea en detrimento de las periféricas menos competitivas (Rodríguez-Pose \& Petrakos, 2004). Tales asimetrías se expresan, en términos sociales y políticos, en "la crisis del proyecto europeo", en "una geografía en crisis" y en el "movimiento de las plazas" y el "levantamiento europeo" (Observatorio Metropolitano, 2011, pp. 51, 59, 93 y 110); ellas se observan, asimismo, en la configuración de "clústeres de desempleo" a través de los territorios europeos (Puga \& Overman, 2002); y a la vez en las reacciones más o menos eurofóbicas como las del UKIP, Partido por la Independencia del Reino Unido, y de Alternative für Deutschland.

Harvey (2007), rememorando la década de 1930 -"ese torturado preludio (luego de la gran depresión) a una guerra intercapitalista planetaria que cambió (...) la geografía histórica del mundo"-, concluye que "la alianza de clase regional se enfrenta a la funesta opción entre la depresión y (quizá) la revolución en el interior o el enfrentamiento militar en el exterior (la forma suprema de 'solución espacial')" (p. 363). En la actualidad, luego de la crisis global de 2008 y de sus secuelas en la Eurozona, donde varios países no recuperan aún el volumen de producto previo a la crisis, esa opción abre paso a otros escenarios. Hoy puede afirmarse que las relaciones entre el "efecto Estado", sus políticas públicas y la financiarización de origen estatal se traducen frecuentemente en una auténtica "geopolítica financiarizada”, estrategia e instrumento de dominio de las economías más desarrolladas o de competencia entre ellas, indudablemente más eficaz que los antiguos colonialismos y los conflictos bélicos entre potencias.

Estas polarizaciones e inequidades en la experiencia de la Eurozona, iniciada en 1951 como Comunidad Europea del Carbón y del Acero, y luego, en 1957, como Comunidad Económica Europea (CEE), una unión aduanera y de libre comercio entre cuyos propósitos explícitos estaba prestar "ayuda a (sus) regiones más pobres" (Krugman, 2012), contribuyen a comprender por qué la actual Unión Monetaria, contrariamente a tales propósitos solidarios, concluyó con "Eurowinners" y "Eurolosers" (Sinn \& Feist, 1997).

No son pocos los que argumentan que muchos de los problemas de la Eurozona -que representa cerca de un quinto del producto mundial- se resolverían con una unión bancaria y fiscal "necesariamente" complementaria a la sola unión monetaria, avanzando más allá del control de la llamada "troika" integrada por la Comisión Europea, el Banco Central Europeo y el Fondo Monetario Internacional. La mayor regulación inherente desincentivaría el "riesgo moral" clásicamente descrito como la "tragedia de los comunes" (Hardin, 1968), ya que en la actual situación un gobierno deficitario puede beneficiarse a costa de otros fiscalmente más disciplinados, afectando el bien comunitario, análogamente a lo que podría ocurrir, en el ámbito monetario, con un banco cualquiera a costa del Banco Central Europeo (Bagus, 2012).

Así, los dilemas y disyuntivas propios de sistemas financieros más o menos descentralizados o centralizados, y las consecuencias que endosan a los mercados 
"locales" de capital (Klagge \& Martin, 2005), replantean económica y territorialmente -dada la mutante geografía de las finanzas y la banca (Alessandrini, Frattianni \& Zazzaro, 2010) - las opciones de supervisión financiera supranacional y/o de "federalismo fiscal" (Carriére-Swallow \& Claro, 2013).

En síntesis, tanto la evaluación de la historia reciente de integración europea, como la configuración de "superregiones" (Gripaios \& Mangles, 1993) y de otros regionalismos sub y supranacionales, hacen evidente que no bastan nuevas técnicas de análisis regional y que no es suficiente una "econometría espacial" (Moreno \& Vayá, 2002), requiriéndose, en la terminología de Harvey (2007, p. 360) una "geopolítica del capitalismo", y en la de Sheppard (2011, portada), una "economía política geográfica”.

\section{Políticas cambiarias y geografía de divisas}

Luego de la última crisis y de sus efectos globales, la "guerra de divisas" -una intervención política cambiaria o una suerte de guerra fría económica no declarada- se preveía y constataba como una consecuencia político-económica y geopolítica, más o menos directa o indirecta, de las políticas públicas nacionales tanto antirrecesión como pro reactivación, y más específicamente, de aquellas pro competitividad. La "desregulación" cambiaria supranacional, que hacía posible esta "guerra", recordaba nostálgicamente, en el imaginario geopolítico de algunos, el sistema monetario internacional concordado a mediados del siglo XX y conocido como Bretton Woods. Refiriéndose a este, Sassen (2010) señalaba que fue "un intento explícito de organizar un sistema de gobernabilidad internacional” (p. 509), impulsado por los EE.UU. como reacción y solución a las devaluaciones sucesivas en diversos países. Para Bagus (2012), los acuerdos de Bretton Woods surgieron en parte como incentivo a los sectores exportadores estadounidenses tras la gran recesión.

En este contexto, los regímenes cambiarios alternativos, las flotaciones cambiarias y las tasas de cambio flexibles, con sus consecuencias en las balanzas de pagos, se relacionaban y asimilaban con crisis político-cambiarias (Carriére-Swallow \& Claro, 2013). La guerra de divisas, auténtica competencia entre monedas (Huerta de Soto, 2012), en los aún inestables primeros tiempos de la poscrisis se acusaba de distintas maneras. Por una parte, en un yen intencionalmente debilitado, que alteraba la competitividad intraasiática -en especial con Singapur, Corea y Taiwán-, para así ayudar a reactivar una economía japonesa estancada y con deflación. Por otra, en un yuan o renmimbi - "dinero del pueblo", significado elocuente por las implicancias sociales de los tipos de cambio y de las guerras de divisas- depreciado políticamente, ampliada su banda de fluctuación y progresivamente su convertibilidad, lo que afectaba, entre otras divisas, al dólar estadounidense. La guerra de divisas se daba también mediante esta misma moneda, subvaluada por las políticas de la Reserva Federal y sus multimillonarios programas de incentivos monetarios pro reactivación, tal vez con más consecuencias internacionales que locales. Y también, y por omisión -o pasivamente, a través de la homogénea rigidez del euro-, inviabilizando una indispensable mayor competitividad, aunque transitoria y previa a una mayor productividad, para los países "periféricos" de la Eurozona, afectados más 
críticamente por la crisis. Y ciertamente también, en el tiempo actual, la guerra de divisas se manifiesta en la creación de fondos internacionales de estabilización, como el de los países BRICs (Brasil, Rusia, India, China y Sudáfrica), y también en una cascada de devaluaciones sucesivas en muchos países emergentes, cuyas monedas se deprecian como consecuencia de los menores precios de sus commodities, que favorecen a las grandes economías que los demandan. Lo mismo ocurre con la reducción de la inversión externa directa, el egreso o franca fuga de capitales -incluida la venta de acciones, de ETF (Exchange Traded Funds) y la especulación vía carry trade entre monedas- y la revalorización reciente de algunas divisas más tradicionales.

¿Hay derrotados y victoriosos en esta guerra de divisas? Como secuelas de esta poco solidaria "competencia entre monedas", los costos económicos y también los sociales de las crisis se "externalizan" a otras naciones, partiendo por las geográficamente cercanas y, a la postre, comprometiéndolas en mayor o menor medida a todas; se endosan recesiones, paros, pobrezas y conflictos sociales de un país a otro; se enrocan territorialmente los desequilibrios fiscales -incluido el de los EE.UU., con el efecto global del cierre parcial de su gobierno o la reducción de su calificación hecha por Standard \& Poor's-, y los déficits y superávits de pagos se truecan incluso entre hemisferios distintos. La ganancia de competitividad de unos se obtiene a costa de la pérdida en otros, a veces en continentes diferentes; la superación del desempleo en determinadas economías revierte en cesantía en otras latitudes; y, en realista pero lamentable conclusión, las políticas públicas que promueven la guerra de divisas redundan y se concretan en algunos territorios ganadores y muchos perdedores, incluyendo entre estos normalmente a las naciones y sociedades más pobres, con economías más vulnerables.

Según Harvey (2007), "se efectúan movimientos resueltos para exportar la devaluación a otras regiones" y "exportar el desempleo al exterior", refiriéndose a "las políticas diseñadas para exportar la devaluación al Tercer Mundo (...) que ponen en peligro la enorme deuda de dichos países (...) en un caso clásico de desplazamiento geográfico" (p. 361). Más aún, aquí puede hipotetizarse, constatada la "huella geográfica" de la crisis y la cronología de su contagio y consecuencias, que los ciclos económicos se inducen asincrónicamente en el territorio internacional, y que además se transfieren con alternancia geográfica.

Como varios autores, Jones y Ward (2002) destacan que el concepto de crisis va asociado a periodos de destrucción y creación y, siguiendo a Habermas y Hay, señalan que las contradicciones económicas internas pueden ser transitoriamente resueltas mediantes procesos de "desplazamiento", los cuales, de acuerdo con Harvey (1982), pueden ser tanto temporales como espaciales (mediante spatial fixes). Tales desplazamientos afectarían tanto a grupos vulnerables como a regiones vulnerables, haciendo evidentes las dificultades de coordinación tanto dentro como a través de distintas escalas espaciales, en un sistema de relaciones intergubernamentales.

La desigual contienda descrita en los párrafos anteriores incluso se produce entre países asociados por tratados comerciales o integrados en uniones monetarias. Durante la crisis y sostenida recesión de la Eurozona, se pudo constatar que la "pérdida de la tasa de cambio variable como herramienta de política monetaria es otra desventaja” para el sur europeo (Van Overtveldt, 2012, p. 53). Sin duda, 
la posibilidad de devaluar era una importante "ventaja de la moneda propia" para los países (periféricos) de la Unión (Krugman, 2012, p. 182), ya que antes de la moneda común, "los países latinos europeos recurrían a la inflación monetaria y desvalorización de sus monedas para readquirir competitividad internacional (...aun) gravando los salarios reales y encareciendo las importaciones" (Huerta de Soto, 2012, pp. 92-93). Así pues, a los tipos de cambio flexibles propiciados hace más de medio siglo por Milton Friedman (1953), parece haberse impuesto y opuesto una moneda única con tan innegables beneficios como indesmentibles e inequitativos costos.

Todo lo anterior confirma que las consecuencias territoriales de las políticas monetarias - propias de un país o comunes a una unión monetaria como la europeason muy desiguales en las distintas regiones, tanto nacionales cuanto subnacionales, atendidas sus diferencias estructurales. De hecho, esas regiones son análogas o similares a pequeñas economías abiertas sin instrumentos monetarios de los que puedan disponer (Dow \& Rodríguez-Fuentes, 1997.

Además de la experiencia de la eurozona, estudios de otras realidades ilustran, complementan y corroboran las constataciones descritas. Así, por ejemplo, Whitt (1996, p. 1) reflexionaba sobre "the Mexican peso crisis", un caso histórico de fuertes devaluaciones de una moneda nacional con implicaciones en y para el naciente North American Free Trade Agreement (NAFTA); y, en un contexto geográfico y de intercambio comercial similar, Beine y Coulombe (2003) investigaban las consecuencias regionales de la "dolarización" en el caso de Canadá. Interrogantes e inquietudes parecidas podrían ser replicadas y reformuladas hoy en relación con las implicancias territoriales de la eventualidad de una "yuanización" -la moneda china llegó a superar al euro en el comercio internacional-, derivada de la creciente primacía de la economía de China. Asimismo, dado el peso económico del Brasil, tanto a nivel global como especialmente sudamericano, es posible verificar el "efecto vecindario" de los altibajos de su moneda, el real, y la localización de tal efecto, positivo o negativo, en sectores y territorios particulares en los países del subcontinente $y$, por cierto y en primer lugar, entre y al interior de los propios estados brasileños.

Es sabido que las variaciones en los tipos de cambio o en las paridades cambiarias -en rigor, "dis-paridades" - trastocan, en conjunto con la competitividad y, más temprano que tarde, la balanza comercial y la de pagos, la relación de la política cambiaria con la monetaria y, en consecuencia, la del tipo de cambio con la tasa de interés. Con algún desfase, en distintos horizontes temporales, sectorial y territorialmente se reasignan los factores productivos, configurándose así en el largo plazo una nueva geografía económica y social e incluso una nueva demografía, promoviendo y reorientando flujos migratorios y alterando las composiciones por sexo y etarias.

Por consiguiente, es objetable la formulación aespacial de las políticas macroeconómicas, ya que sus precios macro -como el tipo de cambio, la tasa de interés y el nivel salarial- producen impactos y cambios regionales y urbanos acentuadamente diferenciados, resultando ser por lo general más influyentes y determinantes, en términos territoriales, que las inversiones directas y localizadas del sector público; y además, lo que es más grave, con resultados en muchas ocasiones contradictorios con las propias políticas vigentes de desarrollo regional y local. Tales externalidades 
territoriales, que pueden ser positivas o negativas según el sector y la localidad, son en estricto rigor un verdadero "subproducto" -no necesariamente consciente ni deseado- de las políticas y gestión de una autoridad económica o monetaria, frente a las que los responsables gubernamentales y las autoridades locales a cargo del desarrollo territorial no tienen más opción que actuar reactivamente, menos en la acepción de oposición y más en la de adecuación (Daher, 1990).

En un escrito de 1999, Jonas destacaba que es necesario reconocer los efectos socioespaciales indirectos que surgen de las políticas aparentemente aespaciales, los que impactarían determinadas localidades y grupos sociales de manera diferencial. Tales efectos indirectos, según Brenner (2004), conformarían el "espacio estatal" en sentido integral -en contraste con el espacio estatal en sentido estricto, referido a las formas de organización espacial del Estado-, en el cual intervienen las instituciones estatales para regular las relaciones sociales y los procesos socioeconómicos. Ambos espacios, el estricto y el integral, incluyendo los efectos espaciales indirectos de las políticas públicas, interactúan, según el mismo autor, para producir formaciones históricas específicas de la espacialidad del Estado. Más tarde, Sassen (2007) confirma lo ya planteado: "debe ser reconocido (...) que ciertas políticas no urbanas en realidad pueden tener efectos fuertes sobre ciudades y regiones" (p. 10), intuyendo que "el punto crítico es la falta de dimensión espacial de estas políticas" (p. 11).

En una reflexión similar, conceptos como los planteados fueron aplicados y verificados por Escobar y Repetto (1993), quienes, en un estudio de caso con base empírica, investigaron cómo la estrategia de desarrollo chilena, asumida a partir de mediados de la década de los ochenta y basada preferentemente en el desarrollo exportador, afectó las rentabilidades de los sectores transables de cada una de las regiones comparativamente al promedio del país.

En la misma línea indagatoria, Daher (1990) ya había postulado que "la paridad cambiaria afecta diferencialmente la distinta especialización sectorial y la diversa geografía de las exportaciones e importaciones (...) con efectos positivos o negativos en el desarrollo rural o urbano; en los sectores productivos o de servicios; en la sustitución capital-trabajo, el empleo y el comportamiento demográfico; en el consumo, el ahorro y la inversión" (pp. 457-458). Este autor especificaba que, de acuerdo con la realidad de la experiencia chilena, por lo general el tipo de cambio real alto suele ser prorregional, y el bajo, prometropolitano. También que, en el plano laboral, la paridad alta tiende a favorecer el empleo, en relación con la sustitución capitaltrabajo, en tanto que la baja habitualmente induce el desempleo productivo y la terciarización del empleo. Agregaba que, en términos demográficos y territoriales, el tipo de cambio alto contribuye a frenar las migraciones del campo a la ciudad llegando, en extremo, incluso a revertirlas; mientras que el bajo las promueve, incrementando la concentración tanto urbana como, especialmente, la metropolitana. Argumentaba, en fin, que en términos sociales, el tipo de cambio bajo promueve el consumo antes que la producción, y tiende a ser regresivo en términos redistributivos; el alto, por el contrario, inhibiría el consumo y favorecería el ahorro junto a la inversión y la producción, tendiendo a ser más distributivo socialmente. Por cierto, las observaciones anteriores son dependientes, más allá de la política cambiaria, de las demás políticas macro, tales como la laboral, tributaria y arancelaria. 
A propósito de las afirmaciones anteriores, la investigación con respaldo estadístico para el caso chileno, desarrollada por Escobar y Repetto (1993), categóricamente verificaba que el tipo de cambio regional relevante es dispar entre las regiones, ya que tanto la estructura productiva específica de cada región, cuanto la composición de socios comerciales para su particular especialización exportadora y también la composición de los países competidores para su sector sustituidor de importaciones, difieren de la nacional.

Ciertamente, en relación con los planteamientos expuestos, es conceptual e históricamente ilustrativo el conocido "síndrome holandés", atendidas sus implicaciones sectoriales y también sus consecuencias territoriales. En efecto, y como es sabido, el descubrimiento en Holanda de yacimientos de gas generó un crecimiento explosivo de la inversión externa directa y, consecuentemente, del ingreso de divisas, apreciándose por consiguiente la moneda local y, por ende, perdiéndose competitividad en las exportaciones de los demás sectores $-\mathrm{y}$, cabe acotar, de sus respectivos territorios y localidades-. La misma apreciación monetaria indujo una baja en el costo de las importaciones, un menor precio de los bienes no transables, y un crecimiento más dinámico tanto del comercio como de los servicios, y también de la construcción. A los cambios anteriores se sumó, por similares razones, un proceso de desindustrialización (Corden \& Neary, 1982). Las transformaciones urbanas inducidas por las alteraciones descritas en los precios relativos no son de difícil deducción, atendida la localización geográfica habitual de los sectores secundario y terciario.

El síndrome descrito ha sido también analizado en relación con la experiencia chilena y su fuerte e histórica dependencia de la exportación de recursos naturales (Álvarez \& Fuentes, 2006). En particular, la "enfermedad holandesa" ha sido referente también para Pereira, Ulloa, O’Ryan y De Miguel (2009), quienes la han asociado al estudio de la altísima y sostenida concentración del sector exportador en el cobre (reconocido como "el sueldo de Chile" y "la viga maestra de [su] economía”), a la que debe agregarse la alta concentración en su mercado (China, en especial), lo que redunda en una mayor vulnerabilidad que debe ser atendida en las políticas gubernamentales. En el caso de Chile, los distintos sectores exportadores, y en especial la gran minería, tienen una localización geográfica latitudinalmente muy definida, lo que permite una clara inteligibilidad del correlato territorial, regional y urbano, del "síndrome chileno".

Las consecuencias del "síndrome holandés" y de las alteraciones en los tipos de cambio reales -sectoriales y regionales, ambos interrelacionados por la especialización económica de los distintos territorios-, en el empleo y en la producción de commodities primarios, y más dramáticamente en los recursos humanos y naturales, generan, a su vez, exposiciones al riego y diversas condiciones de vulnerabilidad. Estas, sin duda, pueden afectar la sustentabilidad socio-laboral, demográfica e incluso ambiental de muchas economías emergentes, y también la de su soporte urbano, radicado en la estructura de asentamientos humanos a escala local, regional y nacional. 


\section{Conclusiones y desafíos}

Este artículo ha tenido por objetivo reflexionar sobre los efectos socioterritoriales de las políticas públicas, sobre todo macroeconómicas, en especial las de aquellas implementadas nacional o supranacionalmente en el período poscrisis. La tesis central, es decir, la afirmación de la no neutralidad espacial de las políticas macro, se postula ciertamente como válida más allá de los episodios de crisis y en cualquier otro escenario económico o histórico, aunque desde luego en el contexto actual tal planteamiento resulta más evidente y crítico.

La globalización, la financiarización y la alta interdependencia que conllevan, hacen más o menos obvio que las medidas de política, sobre todo en el ámbito económico-financiero, tienen efectos relativamente instantáneos y universales, mayores aún si aquellas se originan en los países o uniones de países con mercados más relevantes. Es menos conocido y definitivamente menos obvio el efecto territorial y social de tales políticas: resulta imperiosa, por ende, una agenda de investigación que asuma su complejidad conceptual y los desafíos de implementación práctica de políticas de compensación derivadas de ella.

De hecho, una nueva geografía global emerge como consecuencia no solo de la magnitud de la crisis, sino también de las políticas públicas que intentaron enfrentarla y superarla, algunas a veces reactivamente a la financiarización privada que estuvo en el origen de la crisis, asumiendo el perfil y las características -y también los riesgos- de una creciente y poderosa "financiarización pública".

La nueva arquitectura global del territorio, resultante de las políticas macroeconómicas, específicamente fiscales, monetarias y cambiarias, demuestra su no neutralidad geográfica o social, haciendo evidente la necesidad de evaluar, prever, mitigar y, en extremo, compensar sus efectos inequitativos e impactos discriminatorios en los distintos sectores y zonas. En particular, en tiempos de crisis, la experiencia histórica reciente verifica la necesidad urgente de "territorializar" las políticas de austeridad: si sus costos son altos para toda una nación, suelen ser mucho más agudos en determinados sectores y áreas geográficas, y más dramáticos -en empleo, ingresos, seguridad social y supervivencia- para los más pobres y vulnerables, territorialmente localizables cuando no nítidamente segregados en ciudades y periferias sub e infraurbanas. Las mismas políticas de austeridad, cuando afectan a las grandes economías, contagian sus efectos negativos a las menores y más dependientes, endosándoles muchos de los costos económicos y sociales de crisis y recesiones ajenas a su responsabilidad. Así pues, sea al interior de un mismo país y sociedad, sea entre países y naciones diferentes, tales políticas redistribuyen social y territorialmente los costos recesivos. Y no lo hacen precisamente de manera equitativa.

Otro tanto se concluye respecto de las políticas procompetitividad -incluidas especialmente las guerras de divisas- y sus consecuencias socioterritoriales. En tiempos de crisis, incentivar la competitividad podría parecer acertado e incluso obvio. En esos mismos tiempos, sin embargo, se esperaría una mayor solidaridad internacional, y también nacional, con los sectores menos competitivos. Las heterogéneas consecuencias sociales y territoriales de las políticas cambiarias, al interior del país que las implementa, y las propias de la guerra de divisas entre países, no 
previstas ni menos dimensionadas, pueden terminar opacando las políticas sociales de esos mismos países y minimizando o haciendo menos eficaces las políticas de cooperación y ayuda internacional.

Atenta consideración debe prestarse a las consecuencias, las externalidades o "subproductos" presuntamente inintencionales e inadvertidos de las políticas públicas propias de los gobiernos de los países con economías más desarrolladas, o de los organismos supranacionales en que ellos tienen un peso significativo. Esto, sin embargo, no es suficiente. Especial atención merecen, además, sus recurrentes estrategias de "geopolítica financiarizada" - valga la denominación- formuladas muchas veces con objetivos más o menos explícitos de dominio económico sobre países emergentes y/o de competencia entre potencias, con sus consiguientes impactos sectoriales y territoriales. La reasignación y reorientación geográfica de los flujos e inversiones de capitales, como asimismo la redistribución socioespacial -internacional y subnacional- de riqueza y pobreza inducida por tales políticas merecen también un seguimiento pormenorizado.

En similar perspectiva, la comunidad internacional y sus organismos supranacionales deben ser especialmente sensibles y proactivos frente a la externalización -siempre como consecuencia de las políticas públicas descritas- de muchos de los costos de la crisis a los gobiernos y sociedades de los países emergentes, cuyas autoridades monetarias deben actuar reactivamente sea para defender la devaluación de sus monedas, sea para evitar su sobreapreciación. Ello considerando que se trata de un endoso que se traduce en verdaderos swaps de sustentabilidad para unos pocos países, los más fuertes, a cambio de vulnerabilidad para muchos otros, los más débiles.

La "territorialización" de las políticas de austeridad y el análisis pormenorizado de sus externalidades geográficas entre países y entre regiones es parte ineludible de los desafíos de la agenda de investigación propuesta. Análoga prioridad debe tener el estudio en profundidad de las "externalidades" -en este caso más intencionales y en extremo expresamente planteadas como objetivos- de las políticas pro competitividad, tanto internacionales como locales.

Otra dimensión de la agenda debe atender el estudio de la distribución territorial de la deuda externa pública, según la localización de los países acreedores y deudores, cuya asimetría de corto y largo plazo incide también en la geografía del riesgo país y de los riesgos sub y supranacionales, y con ello ciertamente en el contagio geográfico, por "efecto barrio", de las propias crisis.

Complementariamente, el autor de este artículo ya investiga la geografía de los fondos soberanos, invertidos o "colocados" con una relativa y a veces tácita diversificación intersectorial e internacional, lo que se traduce en aportes de recursos estatales (es decir, "sociales") al crecimiento económico en territorios extranacionales específicos: varios billones de dólares de decenas de países emergentes están en activos internacionales. Esto no es sino otra manifestación y dimensión de la no siempre analizada y cuantificada relación entre políticas públicas y territorio, más relevante aún si se atiende su variabilidad -y, en extremo, volatilidad-geográfica, según se alteren los mercados como resultado, entre otros factores, de las políticas públicas ya mencionadas. 
En el ámbito de las economías en desarrollo o emergentes, en particular en América Latina, y en relación con los ciclos económicos y con las políticas públicas asociadas a ellos, una prioridad de la agenda de investigación y de su aplicación práctica, de singular actualidad y relevancia para los países con economías primarioexportadoras, se vincula a los ciclos boom-bust de sus particulares recursos naturales. Por ende, también lo hace con la históricamente experimentada volatilidad territorial de sus respectivas "regiones-commodities" y, al interior de estas, de sus "ciudadescommodities". Son conocidas las significativas implicaciones de estas coyunturas para las correspondientes economías nacionales, que se manifiestan en alteraciones en sus paridades cambiarias, sus balanzas tanto comerciales como de pagos, y también en sus presupuestos fiscales y en sus mercados laborales, entre otras dimensiones. Pero es necesario discernir más específicamente los efectos directos sobre los sectores y territorios subnacionales de exportación, los que terminan, más temprano que tarde, contaminando las economías nacionales en su conjunto y contagiando sus consecuencias a países enteros. Investigar en profundidad esta compleja dialéctica escalar, no lineal ni simétrica, y menos aún sincrónica, de transferencia territorial de externalidades e incluso de contagio de crisis entre países y entre regiones, es parte de los desafíos pendientes.

Las crisis, y las políticas que las acompañan, se expresan también en procesos más específicos, de gran relevancia económica y social, los que resultan agudizados por aquellas. Entre tales procesos están los que se observan crecientemente en los mercados cada vez más globales de trabajo. En ellos, los diferenciales de salarios reales a nivel internacional determinan -junto a otros factores- las migraciones no solo de grandes multitudes e incluso de grupos etarios (como la expulsión masiva de juventud española por su tasa de paro superior al 50\%), sino también de las industrias manufactureras de la maquila trasnacional.

Asimismo, y siempre en el ámbito social, las consecuencias de una suerte de políticas de "desestatización del Estado de Bienestar", exigidas por la crisis, los desequilibrios fiscales y la presión de organismos financieros internacionales, se expresan como la emergencia de nuevas vulnerabilidades y fragmentaciones sociales; y se constatan tangiblemente como "nuevas pobrezas", presentes en regiones y ciudades donde las desigualdades territoriales poscrisis confirman la inequitativa distribución social y espacial de los costos de tales políticas.

En síntesis, se concluye como cuestión central la inminente necesidad de que los gobiernos y organismos supranacionales evalúen las externalidades socioterritoriales -en caso alguno marginales- de sus políticas públicas postcrisis. Tal evaluación haría posible su reformulación o ajuste, atendidas las consecuencias de tales "subproductos", o al menos su complementación con políticas compensatorias y medidas de prevención y mitigación de sus múltiples y graves impactos sociales y territoriales. 


\section{Referencias bibliográficas}

Aalbers, M. (2009). The globalization and Europeanization of mortgage markets. International Journal of Urban and Regional Research, 33(2), 389-410. doi: 10.1111/j.14682427.2009.00877.x

Aalbers, M. (2012). European mortgage markets before and after the financial crisis. En M.Aalbers (Ed.), Subprime Cities. The political economy of mortgage markets (pp. 120150). Malden, MA: Wiley-Blackwell.

Aglietta, M. (2012). The European vortex. New Left Review, (75), 15-36. Disponible en http:// newleftreview.org/II/75/michel-aglietta-the-european-vortex

Alessandrini, P., Fratianni, M. \& Zazzaro, A. (Eds.). (2010). The changing geography of banking and finance. Nueva York: Springer.

Álvarez, R. \& Fuentes, J. R. (2006). El "síndrome holandés": teoría y revisión de la experiencia internacional. Economía Chilena, 9(3), 97-108. Disponible en http://dialnet.unirioja. es/servlet/articulo?codigo $=2212594$

Bagus, Ph. (2012). A tragédia do Euro. Campinas, sP: Vide Editorial.

Banco Mundial (2008). Informe sobre el desarrollo mundial 2009. Una nueva geografía económica. Washington D.C.: Banco Mundial. Disponible en http://bit.ly/Vutms0

Beine, M. \& Coulombe, S. (2003). Regional perspectives on dollarization in Canada. Journal of Regional Science, 43(3), 541-569. doi: 10.1111/1467-9787.00310

Bravo, H., García, C., Mies, V. \& Tapia, M. (2003). Heterogeneidad de la transmisión monetaria: efectos sectoriales y regionales. Economía Chilena, 6(3), 5-26. Disponible en http://dialnet.unirioja.es/servlet/articulo?codigo $=786721$

Brenner, N. \& Theodore, N. (2002a). Preface: From the new localism to the spaces of neoliberalism. En N. Brenner \& N. Theodore (Eds.), Spaces of neoliberalism .Urban restructuring in North America and Western Europe (pp. v-ix). Malden, MA: Blackwell.

Brenner, N. \& Theodore, N. (2002b). Cities and the geographies of "actually existing neoliberalism". En N. Brenner \& N. Theodore (Eds.), Spaces of neoliberalism .Urban restructuring in North America and Western Europe (pp. 2-32). Malden, MA: Blackwell.

Brenner, N. (2004). New State Space. Urban governance and the rescaling of statehood. Nueva York: Oxford University Press.

Canova, F. (2001). Are EU policies fostering growth and reducing regional inequalities? Els Opuscles del CREI (8), 1-35. Disponible en http://crei.cat/files/ filesOpuscle/17/090429181636_ENG_op8ang.pdf

Carriére-Swallow, Y. \& Claro, S. (2013). Cueste lo que cueste. La batalla por salvar el euro y Europa. Santiago: Ediciones Pontificia Universidad Católica de Chile.

Corden, W. \& Neary, J. P. (1982). Booming sector and the de-industrialisation in a small open economy. Economic Journal, 92(368), 825-848. doi: 10.2307/2232670

Corpataux, J. \& Crevoisier, O. (2005). Increased capital mobility/liquidity and its repercussions at regional level. Some lessons from the experiences of Switzerland and the United Kingdom (1975-2000). European Urban and Regional Studies, 12(4), 315-334. doi: $10.1177 / 0969776405058945$

Corpataux, J., Crevoisier, O. \& Theurillat, T. (2009). The expansion of the finance industry and its impacts on the economy: A territorial approach based on Swiss pension funds. Economic Geography, 85(3), 313-334. doi:10.1111/j.1944-8287.2009.01035.x 
Cuadrado-Roura, J. R. (2014). ¿Es tan "nueva” la "Nueva Geografía Económica”? EURE, 40(120), 5-28. http://dx.doi.org/10.4067/S0250-71612014000200001

Daher, A. (1990). Políticas macroeconómicas, tipo de cambio y desarrollo territorial. Escenarios chilenos. En F. Alburquerque; C. de Mattos \& R. Jordán (Eds.), Revolución tecnológica y reestructuración productiva: impactos y desafios territoriales (pp. 457-478). Buenos Aires: ILPES/ONU, IEU/PUC, GeL.

Daher, A. (2003). Regiones-commodities. Crisis y contagio en Chile. EURE, 29(86), 89-108. http://dx.doi.org/10.4067/S0250-71612003008600005

Daher, A. (2013a). El sector inmobiliario y las crisis económicas. EURE, 39(118), 47-76. http://dx.doi.org/10.4067/S0250-71612013000300003

Daher, A. (2013b). Territorios de la financiarización urbana y de las crisis inmobiliarias. Revista de Geografia Norte Grande, (56), 7-30. http://dx.doi.org/10.4067/S071834022013000300002

Dow, Sh. \& Rodríguez-Fuentes, C. (1997). Regional finance: A survey. Regional Studies, 31(9), 903-920. doi: 10.1080/00343409750130029

Dymski, G. (2012). Subprime crisis and urban problematic. En M. Aalbers (Ed.), Subprime Cities. The political economy of mortgage markets (pp. 293-314). Malden, MA: WileyBlackwell.

Dymsky, G. (2009). The global financial customer and the spaciality of exclusion after the "end of geography". Cambridge Journal of Regions, Economy and Society, 2(2), 267-285. doi: $10.1093 /$ cjres/rsp011

Escobar, B. \& Repetto, A. (1993). Efectos de la estrategia de desarrollo chilena en las regiones: una estimación de la rentabilidad del sector transable regional. Colección Estudios CIEPLAN 37, 5-36. Disponible en http://www.cieplan.org/media/publicaciones/ archivos/63/Capitulo_1.pdf

Esquivel, G. \& Larraín, F. (1999). América Latina frente a la crisis asiática. Estudios Públicos, 73 , 5-30. Disponible en http://www.cepchile.cl/dms/archivo_1401_762/rev73_esquivellarrain.pdf

Ezcurra, R., Pascual, P. \& Rapún, M. (2003). Movilidad y desigualdad regional en la Unión Europea. Investigaciones Regionales, (2), 5-30. Disponible en http://www.redalyc.org/ articulo.oa?id=28900201

French, Sh., Leyshon, A. \& Thrift, N. (2009). A very geographical crisis: The making and breaking of the 2007-2008 financial crisis. Cambridge Journal of Regions, Economy and Society, 2(2), 287-302. doi: 10.1093/cjres/rsp013

Friedman, M. (1953). The case of flexible exchange rates. En M Friedman, Essays in Positive Economics (pp. 157-203). Chicago, IL: University of Chicago Press.

Gill, S. (1995). Globalisation, market civilisation and disciplinary neoliberalism. Millennium, 24(3), 399-423. doi: 10.1177/03058298950240030801

González, S. (2005). La geografía escalar del capitalismo actual. Scripta Nova, Revista Electrónica de Geografía y Ciencias Sociales, 9(189). Disponible en http://www.ub.edu/geocrit/sn/ sn-189.htm

Gripaios, P. \& Mangles, T. (1993). An analysis of European super regions. Regional Studies, 27(8), 745-757. doi: 10.1080/00343409312331347925

Hardin, G. (1968). The tragedy of the commons. Science New Series, 162(3859), 1243-1248. doi: $10.1126 /$ science.162.3859.1243 
Harvey, D. (1982). The limits of capital. Chicago, IL: University of Chicago Press.

Harvey, D. (2000). Spaces of hope. Edimburgo: Edimburgh University Press.

Harvey, D. (2007). Espacios del capital. Hacia una geografía crítica. Madrid: Akal.

Harvey, D. (2011). O enigma do capital e as crises do capitalismo. São Paulo: Boitempo.

Harvey, D. (2012a). Las raíces urbanas de las crisis financieras. En M. Belil, J. Borja \& M.Corti (Eds.), Ciudades: una ecuación imposible (pp. 321-358). Buenos Aires: Café de las Ciudades.

Harvey, D. (2012b). The urban roots of the financial crisis. En M. Aalbers (Ed.), Subprime Cities. The political economy of mortgage markets (pp. xiii-xix). Malden, MA: WileyBlackwell.

Hernández, L. \& Valdés, R. (2001). What drives contagion: Trade, neighborhood or financial links? (IMF Working Paper / International Monetary Fund WP/01/29). Disponible en https://www.imf.org/external/pubs/ft/wp/2001/wp0129.pdf

Huerta de Soto, J. (2012). Prefácio. En Ph. Bagus, A tragédia do Euro (pp. 11-17). Campinas, sp: Vide Editorial (11-17).

Janoschka, M. \& Hidalgo, R. (2014). La ciudad neoliberal: estímulos de reflexión crítica. En R. Hidalgo \& M. Janoschka (Coords.), La ciudad neoliberal. Gentrificación y exclusión en Santiago de Chile, Buenos Aires, Ciudad de México y Madrid (7-32). Santiago de Chile: LOM.

Jonas, M. (1999). New institutional spaces. Londres: Jessica Kingsley.

Jones, M. \& Ward, K. (2002). Excavating the logic of British urban policy: Neoliberalism as the "crisis of crisis-management". En N. Brenner \& N. Theodore (Eds.), Spaces of neoliberalism. Urban restructuring in North America and Western Europe (pp. 126147). Malden, ma: Blackwell. Artículo publicado en Antipode, 34(3), 473-494. doi: $10.1111 / 1467-8330.00251$

Kennen, P. (1969). The theory of optimum currency areas: an eclectic view. En R. Mundell \& A. Swoboda (Eds.), Monetary problems of the international economy (pp. 41-68). Chicago, IL: University of Chicago Press.

Klagge, B. \& Martin, R. (2005). Decentralized versus centralized financial systems: Is there a case for local capital markets? Journal of Economic Geography, 5(4), 387-421. doi: $10.1093 / \mathrm{jeg} / \mathrm{lbh} 071$

Krugman, P. (2010). De vuelta a la economía de la gran depresión y la crisis del 2008. Bogotá: Norma.

Krugman, P. (2012). ¡Acabemos ya con esta crisis! Barcelona: Crítica.

Lee, R., Clark, G., Pollard, J. \& Leyshon, A. (2009). The remit of financial geography - before and after the crisis. Journal of Economic Geography, 9(5), 723-747. doi: 10.1093/jeg/ lbp035

Leyshon, A. (1997). Money / Space: Geography of monetary transformation. Londres: Taylor and Francis.

Martin, R. \& Minns, R. (1995). Undermining the financial basis of regions. Regional Studies, 29(2), 125-144. doi: 10.1080/00343409512331348853

Martin, R. (1998). Money and the space economy. Hoboken, NJ: Wiley.

Martin, R. (2011). The local geographies of the financial crisis: from the housing bubble to economic recession and beyond. Journal of Economic Geography, 11(4), 587-618. doi: $10.1093 /$ jeg/lbq024 
McKinnon, R. (1963). Optimum currency areas. American Economic Review, 53(4), 717-725. Disponible en http://bit.ly/1nCJS8c

Méndez, R. (2013). Impactos de la crisis internacional en la Región Metropolitana de Madrid. En J. Noyola, C. de Mattos \& A. Orellana (Eds.), Urbanización en tiempos de crisis. Impactos, desafios y propuestas (pp. 45-72). Santiago: Colección Estudios Urbanos, Pontificia Universidad Católica de Chile.

Moreno, R. \& Vayá, E. (2002). Econometría espacial: nuevas técnicas para el análisis regional. Una aplicación a las regiones europeas. Investigaciones Regionales, (1), 83-106. Disponible en http://www.redalyc.org/articulo.oa?id=28900104

Mundell, R. (1961). A theory of optimum currency areas. American Economic Review, 51(3), 657-665. Disponible en https://www.aeaweb.org/aer/top20/51.4.657-665.pdf

Noyola, J., de Mattos, C. \& Orellana, A. (eds.) (2013). Urbanización en tiempos de crisis. Impactos, desafios y propuestas. Santiago: Colección Estudios Urbanos uc.

O’Brien R. (1992). Global financial integration: the end of geography. Londres: Chatham House Papers.

Observatorio Metropolitano (2011). Crisis y revolución en Europa. People of Europe, rise up. Madrid: Traficante de Sueños. Disponible en http://www.rebelion.org/docs/138745.pdf Olivera, P. (2014). Neoliberalismo en la Ciudad de México: polarización y gentrificación. En R.Hidalgo \& M. Janoschka (Coords.), La ciudad neoliberal. Gentrificación y exclusión en Santiago de Chile, Buenos Aires, Ciudad de México y Madrid (pp. 151-178)). Santiago de Chile: LOM.

Peck, J. \& Tickell, A. (2002). Neoliberalizing space. En N. Brenner \& N. Theodore (Eds.), Spaces of neoliberalism. Urban restructuring in North America and Western Europe (pp. 33-57). Malden, MA: Blackwell. Artículo publicado Antipode, 34(3), 380-404. doi: 10.1111/1467-8330.00247

Pereira, M., Ulloa, A., O’Ryan, R. \& De Miguel, C. (2009). Sindrome holandés, regalías mineras y politicas de gobierno para un pais dependiente de recursos naturales: el cobre en Chile. Serie Medio Ambiente y Desarrollo 140. Santiago: Comisión Económica para América Latina y el Caribe (Cepal). Disponible en http://repositorio.cepal.org/bitstream/ handle/11362/5681/S0900767_es.pdf?sequence $=1$

Perlo, M. (2011). Cities in times of crisis: The response of local governments in light of the global economic crisis (IURD Working Paper 2011-01). Berkeley, CA: University of CaliforniaBerkeley, Institute of Urban and Regional Development (IURD). Disponible en http:// escholarship.org/uc/item/3432p4rb\#page-1

Puga, D. \& Overman, H. (2002). Unemployment clusters across Europe's regions and countries. Economic Policy, 17(34), 115-147. http://dx.doi.org/10.1111/1468-0327.00085

Reinhart, C. y Rogoff, K. (2011). Esta vez es distinto: ocho siglos de necedad financiera. México: FCE.

Rodil, O. (2008). La competitividad y su impacto sobre el crecimiento económico regional de la Unión Europea (1995-2004). Revista de Estudios Regionales, (83), 49-78.

Rodríguez, C. (2005). Un análisis comparado de la inversión extranjera directa efectiva y potencial de las regiones españolas. Revista de Estudios Regionales, (73), 13-42. Disponible en http://www.redalyc.org/pdf/755/75507301.pdf

Rodríguez-Pose, A. \& Petrakos, G. (2004). Integración económica y desequilibrios territoriales en la Unión Europea. EURE, 29(89), 63-80. http://dx.doi.org/10.4067/S025071612004008900004 
Roubini, N. \& Mihm, S. (2010). Cómo salimos de esta. Santiago: Destino.

Sala-i-Martin, X. \& Sachs, J. (1991). Fiscal federalism and optimum currency areas: evidence for Europe from the United States (nBER Working Papers 3855). Cambridge, MA: National Bureau of Economic Research.

Sassen, S. (1998). As cidades na economia mundial. São Paulo: Studio Nobel.

Sassen, S. (2001). ¿Perdiendo el control? La soberanía en la era de la globalización. Barcelona: Bellaterra.

Sassen, S. (2003). Contrageografías de la globalización. Género y ciudadanía en los circuitos transfronterizos. Madrid: Traficantes de Sueños. Disponible en http://bit.ly/1VvtTmG

Sassen, S. (2007). El reposicionamiento de las ciudades y regiones urbanas en una economía global: ampliando las opciones de políticas y gobernanza. EURE, 33(100), 9-34. http://dx.doi.org/10.4067/S0250-71612007000300002

Sassen, S. (2010). Territorio, autoridad y derechos. De los ensamblajes medievales a los ensamblajes globales. Buenos Aires: Katz.

Sassen, S. (2012). Expanding the terrain for global capital: When local housing becomes an electronic instrument. En M. Aalbers (Ed.), Subprime Cities. The political economy of mortgage markets (pp. 74-96). Malden, MA: Wiley-Blackwell.

Sheppard, E. (2011). Geographical political economy. Journal of Economic Geography, 11(2), 319-331. doi: 10.1093/jeg/lbq049

Sinn, H-W. \& Feist, H. (1997). Eurowinners and Eurolosers: The distribution of seigniorage wealth in EMU. European Journal of Political Economy, 13(4), 665-689. http://dx.doi. org/10.1016/S0176-2680(97)00029-3

Sokol, M. (2013). Towards a "newer" economic geography? Injecting finance and financialisation into economic geographies. Cambridge Journal of Regions, Economy and Society, 6(3), 501-515. doi: $10.1093 /$ cjres/rst022

Soros, G. (2012). Financial turmoil in Europe and the United States. Nueva York: Public Affairs.

Stiglitz, J. (2010). El libre mercado y el hundimiento de la economía mundial. Buenos Aires: Taurus.

Suárez-Villa, L. \& Cuadrado Roura, J. R. (1993). Regional economic integration and the evolution of disparities. Papers in Regional Science, 72(4), 369-387. doi: 10.1111/ j.1435-5597.1993.tb01883.x

Van Overtveldt, J. (2012). O Fim do Euro: A história da moeda da União Europeia e seu futuro incerto. Rio de Janeiro: Elsevier.

Wainwright, Th. (2012). Building new markets: Transferring securitization, bond-rating, and a crisis from the us to the UK. En M. Aalbers (Ed.), Subprime Cities. The political economy of mortgage markets (pp. 97-119). Malden, MA: Wiley-Blackwell.

Whitt, J. (1996).The Mexican peso crisis. Economic Review, 81(1), 1-20. Disponible en http:// bit.ly/1SoeMfl 